\title{
O papel profissional do enfermeiro no Sistema Único de Saúde: da saúde comunitária à estratégia de saúde da família
}

\author{
The role of the nurse in the Brazilian Unified Heath System: \\ from community health to the family health strategy
}

Dirce Stein Backes ${ }^{1}$

Marli Stein Backes ${ }^{2}$

Alacoque Lorenzini Erdmann ${ }^{2}$

Andreas Büscher ${ }^{3}$

${ }^{1}$ Conselho de Áreas, Centro

Abstract The scope of this paper is to take a retrospective look at the professional role of the nurse in the Brazilian Unified Health System and understand the meaning of their social practice in this field of discussions and theoretical-practical meanings. The Grounded Theory was used as a methodological reference and interviews were the technique for data collection, conducted between May and December of 2007, with 35 health professionals and others. The codification and data analysis revealed that the Unified Health System and especially the Family Health Strategy, should be considered facilitating and stimulating strategies of the process of expansion and consolidation of nursing care as a social entrepreneurial practice, since they pave the way to a new approach in community intervention, through seeing the human being as a multi-dimensional and singular being, inserted in a real and concrete context. Key words Nurse's role, Community health nursing, Public health, Unified Health System, Family Health Program
Resumo Objetiva-se, com este trabalho, possibilitar um olhar retrospectivo do papel profissional do enfermeiro no Sistema Único de Saúde brasileiro, bem como compreender o significado de sua prática social neste campo de discussões e significações teórico-práticas. Utilizou-se como referencial metodológico a Grounded Theory e como técnica de coleta de dados a entrevista, realizada entre maio a dezembro de 2007, com 35 profissionais da saúde e outros. A codificação e análise dos dados evidenciaram que o Sistema Único de Saúde e, de modo especial a Estratégia Saúde da Família, podem/devem ser considerados estratégias facilitadoras e estimuladoras do processo de ampliação e consolidação do cuidado de enfermagem como prática social empreendedora, à medida que sinalizam para uma nova abordagem de intervenção comunitária, pela valorização do ser humano como um ser singular e multidimensional, inserido em seu contexto real e concreto.

Palavras-chave Papel do enfermeiro, Enfermagem em Saúde Comunitária, Saúde coletiva, Sistema Único de Saúde, Programa Saúde da Família 


\section{Introdução}

A enfermagem vem ampliando, a cada dia, o seu espaço na área da saúde, tanto no contexto nacional quanto no cenário internacional. O enfermeiro assume um papel cada vez mais decisivo e próativo no que se refere à identificação das necessidades de cuidado da população, bem como na promoção e proteção da saúde dos indivíduos em suas diferentes dimensões. O cuidado de enfermagem é, portanto, um componente fundamental no sistema de saúde local, que apresenta os seus reflexos a nível regional e nacional e, por isso, também motivo de crescentes debates e novas significações.

Mesmo que interligada e complementada por outros saberes profissionais, a enfermagem pode ser amplamente definida como a ciência do cuidado integral e integrador em saúde, tanto no sentido de assistir e coordenar as práticas de cuidado, quanto no sentido de promover e proteger a saúde dos indivíduos, famílias e comunidades. Nessa direção, o cuidado de enfermagem configura-se como prática social empreendedora, pela inserção ativa e pró-ativa nos diferentes espaços de atuação profissional e, principalmente, pelas possibilidades interativas e associativas com os diferentes setores e contextos sociais ${ }^{1}$.

Evidências internacionais acenam para a importância do papel profissional do enfermeiro na saúde coletiva, tanto no espaço domiciliar quanto no espaço comunitário ou nos centros de saúde comunitários. A enfermagem tem a possibilidade de operar, de forma criativa e autônoma, nos diferentes níveis de atenção à saúde, seja através da educação em saúde, seja na promoção ou na reabilitação da saúde dos indivíduos. Esse processo se dá, particularmente, no esforço pelo levantamento de situações críticas e a intervenção sistematizada de um plano de cuidados, capaz de superar as fragmentações e assegurar a continuidade e a resolutividade do cuidado em saúde ${ }^{2-4}$. Nesse campo de discussões, o papel profissional do enfermeiro é ampliado pela estratégia da Organização Mundial da Saúde "Saúde 21", saúde para todos no século 21 e prioridade número um para a região européia, a qual se concentra em alcançar níveis cada vez mais amplos de saúde e, desse modo, favorecer ao ser humano uma vida social e economicamente produtiva e com mais qualidade ${ }^{5}$.

No Brasil, vários estudiosos se empenham em dar visibilidade ao papel profissional do enfermeiro, seja como prática social comunitária, autônoma, ou como prática assistencial institu- cionalizada $^{6-10}$. É preciso levar em conta, no entanto, que a enfermagem como prática comunitária se cunhou de novos significados conceituais, possibilitados pela concepção de saúde coletiva, campo ainda em constituição e que, crescentemente, vem assumindo diversas formas e abordagens.

O termo saúde coletiva surgiu, mais especificamente, no fim da década de 70, em um momento de reordenamento de um conjunto de práticas assistenciais, diante da necessidade de ampliar a compreensão do processo saúde-doença dos indivíduos e comunidades, pela inserção e valorização dos diferentes saberes profissionais e a integração com os diferentes setores sociais. A compreensão do coletivo significa, a partir de então, a apreensão do individual em seu contexto estruturado de práticas sociais. Significa reconhecer o indivíduo - ser individual - como um ser social, em constante interação com os outros indivíduos e com o seu entorno, ou seja, o indivíduo se transforma e é transformado continuamente, por meio das relações e interações, tornando-se protagonista e autor do processo saúde-doença em seu contexto real e concreto ${ }^{11}$.

A mudança conceitual do termo - saúde pública para saúde coletiva - é reflexo de um intenso engajamento dos movimentos sociais na luta pela democratização do país, além da centralidade assumida pela Assembléia Nacional Constituinte, em 1977. Emergida como parte dessa luta pela democracia, a Reforma Sanitária, no Brasil, alcançou a garantia constitucional do direito universal à saúde e a construção institucional do Sistema Único de Saúde (SUS), aprovado na Constituição Federal de $1988^{12,13}$.

\section{O Sistema Único de Saúde - possibilidades profissionais}

O SUS foi criado, nessa perspectiva, a partir das manifestações de um conjunto de necessidades sociais de saúde, as quais imprimem um caráter ético-moral que a defende como direito de todo cidadão. Enquanto conquista das lutas participativas e democráticas, o SUS se desenvolve com base nos princípios de acesso, universalidade, equidade e integralidade, e com base nas diretrizes organizativas de descentralização, regionalização, hierarquização e participação da comunidade ${ }^{14}$.

Como estratégia de reformulação do modelo brasileiro de atenção à saúde e o fortalecimento dos princípios e diretrizes do SUS, o Ministério da Saúde criou, em 1994, a Estratégia Saúde da Família (ESF), inicialmente denominada Pro- 
grama de Saúde da Família. A estratégia nasceu na tentativa de repensar os padrões de pensamento e comportamento dos profissionais e cidadãos brasileiros, até então vigentes. Sistematizada e orientada por equipes de saúde da família que envolve médicos, enfermeiros, técnicos de enfermagem, odontólogos e Agentes Comunitários de Saúde (ACS), a ESF busca discutir e ampliar o tradicional modelo sanitário médico-curativista, para a compreensão de uma abordagem coletiva, multi e interprofissional, centrada na família e na comunidade, inserida em seu contexto real e concreto ${ }^{8,9}$.

Sendo a ESF uma estratégia de fomento à participação da população, esta deve, crescentemente, promover uma nova relação entre os sujeitos, onde tanto o profissional quanto o usuário podem/devem ser produtores e construtores de um viver mais saudável. Este envolvimento, no entanto, só é possível mediante um processo dialógico entre os diferentes saberes, no qual cada um contribui com o seu conhecimento peculiar e juntos possibilitam uma interação efetiva pela valorização das diferentes experiências e expectativas de vida9.

É neste contexto de discussões, conquistas e desafios que o enfermeiro precisa delinear cada vez mais e melhor o seu campo de atuação profissional e desenvolver o seu projeto político-legal, coerente com os princípios e diretrizes do SUS, bem como com as diretivas da ESF. Um projeto profissional, portanto, que necessita considerar o ser humano - ser individual e coletivo como sujeito e ator social. Nessa direção, questiona-se: Qual o significado do papel profissional do enfermeiro no Sistema Único de Saúde brasileiro e qual o significado de sua prática social neste campo de discussões e significações teórico-práticas?

Pretende-se, a partir do exposto, situar a enfermagem frente às práticas em saúde coletiva, mais especificamente no SUS, para compreender como esta vem se constituindo enquanto uma das disciplinas que contribui de forma decisiva para a consolidação dos princípios e diretrizes do SUS. Objetiva-se, assim, possibilitar um olhar retrospectivo sobre o papel profissional do enfermeiro no Sistema Único de Saúde brasileiro, bem como compreender o significado de sua prática social neste campo de discussões e significações teórico-práticas.

\section{Metodologia}

Trata-se de um estudo de abordagem qualitativa-exploratória, orientado pelo método Grounded Theory.

A Grounded Theory é um método que permite explorar os dados de forma criativa, abrangente e interativa, por levar em conta que muitas coisas podem ser consideradas dados de pesquisa. Constituiu-se num processo em que os dados coletados, codificados e comparados de forma simultânea e sistemática, possibilitam explorar os significados e hipóteses, nesse caso, do papel do enfermeiro, sob diferentes ângulos e espaços ${ }^{15,16}$.

A amostra teórica foi constituída por 35 (trinta e cinco) participantes, distribuídos em 04 (quatro) grupos amostrais de 08 (oito) ou 09 (nove) componentes, inseridos nos mais diferentes cenários da saúde da Grande Florianópolis/SC, Brasil.

O primeiro grupo foi selecionado pelos pesquisadores de forma intencional. Já o segundo, foi indicado pelos componentes do primeiro grupo e, assim, sucessivamente, considerando sempre as hipóteses emergentes de cada entrevista analisada e codificada. Dentre os entrevistados, encontram-se: enfermeiros, médicos, odontólogos, nutricionistas, farmacêuticos, psicólogos, teólogos, pedagogos, gestores e usuários da saúde, que após serem orientados acerca dos objetivos da pesquisa, assinaram o termo de consentimento livre e esclarecido.

O processo de coleta e análise dos dados, realizado concomitantemente entre os meses de maio a dezembro de 2007, conforme os pressupostos da Grounded Theory, possibilitou, além da construção de hipóteses, gerar conhecimentos que permitiram ampliar a compreensão do papel do enfermeiro no SUS e na ESF, bem como vislumbrar novas possibilidades empreendedoras na enfermagem.

O projeto foi aprovado pelo Comitê de Ética em Pesquisa da Universidade Federal de Santa Catarina - UFSC. Para manter o sigilo das informações, os participantes da pesquisa foram identificados, ao longo do texto, pela letra "E" (entrevistado), seguida do número correspondente à ordem de realização das entrevistas (p1, p2, p3... p35). 


\section{Resultados}

O SUS é reconhecido e ressaltado pelos entrevistados como um dos maiores sistemas de mobilização social, pelo acesso, cobertura e garantia da continuidade pela integração com os diversos setores e às diversas políticas sociais. É reconhecido igualmente como um sistema empreendedor, por promover a participação da comunidade nas discussões que dizem respeito à saúde, conforme expresso na fala a seguir:

O sistema de saúde do país, talvez seja um dos maiores setores de mobilização social. Se considerarmos empreendedorismo como transformação, mobilização, isto existe, porque produzimos grandes resultados, principalmente na saúde... Os próprios conselhos de saúde que mobilizam a participação da população e o controle social devem ser considerados empreendedores... na lógica do social, os princípios do SUS possuem a definição para o sucesso (E9).

Por meio dos princípios da universalidade, integralidade, equidade, trouxe implícito um novo modelo de intervenção e participação social, especialmente com a criação da Estratégia Saúde da Família, conforme refletem as falas a seguir:

Precisamos reconhecer que o SUS trouxe novas oportunidades de mobilização e participação social, principalmente pelo programa estratégia saúde da família (E3).

O SUS representa para o enfermeiro e demais profissionais da saúde uma grande conquista, pela ampliação das oportunidades de atuação, principalmente na educação e promoção da saúde (E7).

\section{SUS - Ampliação da intervenção comunitário-coletiva}

Para os entrevistados, o SUS também passou a dar um novo sentido à saúde como bem-social, com foco na promoção e intervenção comunitário-coletiva, com vistas a superar a fragmentação do modelo biomédico e hospitalocêntrico, conforme salientam as falas: Anos atrás, o hospital era mais importante porque tinha $70 \%$ de trabalhadores empregados... isto mudou. A lógica hoje é a saúde coletiva (E13).

A ênfase a partir do SUS é a promoção da saúde, a atenção básica, a saúde da família (E16).

[...] temos uma amplitude de sistema que nenhum outro país tem (E17).

O SUS possibilitou um olhar mais ampliado sobre o processo saúde-doença, pela valorização dos diferentes saberes profissionais. Possibilitou compreender o indivíduo - ser singular e multidimensional - em seu contexto real e concreto.

Os entrevistados envidenciaram que, com a criação do SUS, os profissionais da saúde, mais especificamente o profissional enfermeiro, ampliou a sua atuação e inserção no campo comunitário e social. Esta prática foi ampliada ainda mais, com as oportunidades oferecidas pela ESF, a qual proporcionou maior visibilidade e se apresenta como um espaço aberto, sensível e flexível para a emancipação e a transformação social, como bem evidencia a fala, a seguir:

A ESF deu maior visibilidade para o papel profissional do enfermeiro, principalmente no campo da saúde coletiva. O enfermeiro na saúde coletiva sempre existiu... mas hoje está tendo uma maior visibilidade. A ESF se apresenta como um espaço aberto e sensivel para atuar nas famílias, comunidades, associação de moradores, nas escolas, nos sindicatos, nas políticas públicas e outros. Então, hoje, o enfermeiro tem um espaço aberto na sociedade para trabalhar a questão da cidadania, das políticas públicas, da educação em saúde (E27).

\section{SUS - Novo modo de pensar e agir}

Com a criação do SUS, e especialmente a ESF, o enfermeiro tem o seu espaço de atuação garantido, mesmo que para alguns profissionais a emergência do novo modo de pensar e agir ainda represente certo desconforto e insegurança pela necessidade de inserção ativa e responsável na vida das comunidades, como reflete a fala:

Mesmo sem a coragem de mudar, o enfermeiro e os outros profissionais são levados (provocados) pelo sistema de saúde a mudarem os seus comportamentos e o modo de pensar (E9).

Nessa direção, os entrevistados trazem à tona que independente da vontade pessoal/profissional, os enfermeiros, como os demais profissionais da saúde são impulsionados pelo sistema a adotarem uma nova postura de intervenção nos diferentes cenários da saúde:

Mesmo que este profissional não ouse por conta própria, ele está sendo colocado num espaço que está cheio de oportunidades e desafios que precisam ser considerados (E31).

Esse novo modo de pensar e agir, constatado tanto pelos profissionais quanto pelos usuários da saúde, que impregnados pela lógica fragmentada, dicotômica e pouco resolutiva, demonstraram, inicialmente, insegurança e desconforto diante do novo, como evidencia a fala de uma das enfermeiras entrevistadas: 
O usuário da saúde ainda está muito acostumado a ser atendido em partes, então no início ele estranha muito [...]. Ficou bastante visível nas primeiras visitas que o paciente e a família estão desacreditados (E7).

O espaço social, caracterizado como espaço familiar e comunitário, possibilita um aprendizado instigador e contínuo, além de uma intensa troca de experiências, tanto para enfermeiros e outros profissionais como para os usuários da saúde. Possibilita, também, a realização e bemestar profissional, pela conquista da autonomia e reconhecimento por parte dos usuários da saúde, conforme refletem as falas:

Eu preciso encontrar espaços onde eu possa fortalecer a autonomia, e a comunidade me proporciona isto... (E6).

Na família, a gente precisa levar em conta a autonomia do usuário... tem a possibilidade de troca, de eu também aprender. Então existe toda uma interação, o usuário passa a ser ator do processo (E13).

Pelo fato de ser um curso que tem uma formação universitária generalista, a enfermagem está qualificada, no entender dos entrevistados, para integrar e fomentar ativamente os princípios do sistema de saúde vigente, sobretudo, nas atividades interativas e gerenciais, as quais requerem maior envolvimento, sistematização e comprometimento com as reais necessidades de saúde da população.

\section{SUS - Ampliação das interações e associações profissionais}

A partir da lógica do SUS, o cuidado de enfermagem é visibilizado como prática interativa, multidimensional e interdisciplinar, ou seja, como prática social que integra uma rede de relações e associações comunitárias. Nessa direção, os participantes entendem que é preciso ocorrer uma articulação crescente com os diferentes profissionais que atuam no sistema de saúde para que a pessoa humana, em seu contexto singular e coletivo, seja compreendida como um ser integral protagonista do seu processo saúde-doença.

O enfermeiro é reconhecido, nessa perspectiva, pela habilidade interativa e associativa, por compreender o ser humano como um todo, pela integralidade da assistência à saúde, pela capacidade de acolher e identificar-se com as necessidades e expectativas dos indivíduos, pela capacidade de interagir diretamente com o usuário e a comunidade, bem como pela capacidade de promover o diálogo entre os usuários e a equipe de saúde da família. No entender dos entrevistados, o enfermeiro se aproxima, identifica e procura criar uma relação de empatia com o usuário, independentemente das suas condições sociais. O enfermeiro é aquele que encaminha e otimiza as intervenções de cuidado em saúde de modo que integre e contemple tanto os saberes profissionais quanto os saberes dos usuários.

Os resultados evidenciam, em suma, que os profissionais de saúde se encontram, por meio do SUS e mais especificamente através da ESF, diante de um novo modelo assistencial, no qual o impacto do desconhecido e a insegurança diante do novo são inevitáveis, mas motivadores e animadores para o crescimento profissional e o desenvolvimento social integrado e integrador. Desse modo, tanto o SUS quanto a ESF podem/ devem ser considerados estratégias facilitadoras e estimuladoras do processo de ampliação e consolidação do cuidado de enfermagem como prática social empreendedora, comprometida com o desenvolvimento humano e a transformação social.

\section{Discussão}

As discussões em torno da atuação dos profissionais da saúde no SUS, convergem para o reconhecimento de que o enfermeiro é o interlocutor e o principal agente catalisador das políticas e programas voltados para a saúde coletiva, em especial para a ESF que requer um envolvimento efetivo com as reais necessidades de saúde das famílias e comunidades ${ }^{6}$.

Esse pensar é corroborado por profissionais da área, ao argumentarem que o enfermeiro, cuja essência e especificidade é o cuidado do ser humano em todas as suas dimensões, individual ou coletivamente, de forma integral e holística, é formado para atuar nos diferentes espaços sociais, tais como: na atenção, na gestão, no ensino, na pesquisa, no controle social, bem como no fomento de ações educativas e de promoção da saúde dos indivíduos, famílias e comunidades. Nessa direção, a autonomia e o protagonismo social do enfermeiro são construídos por conquistas técnico-científicas, legais e políticas pelo desenvolvimento de práticas cidadãs comprometidas com o bem-estar social ${ }^{17,18,8}$.

O Ministério da Saúde brasileiro, por meio do Departamento de Gestão da Educação na Saúde, vem atuando, crescentemente, no sentido de reorientar e ampliar a formação dos profissionais da saúde, de modo geral, para a área da 
Atenção Primária, com o intuito de fortalecer a cobertura da ESF e aumentar a resolutividade da atenção à saúde no SUS. A referida formação também faz parte da Política Nacional de Educação em Saúde, que traz à tona aspectos como o conceito ampliado de saúde; a utilização de metodologias ativas de ensino-aprendizagem, que considerem o trabalho como eixo estruturante das atividades; o trabalho em equipe multiprofissional e transdisciplinar; a integração entre o ensino e os serviços de saúde; o aperfeiçoamento da atenção integral à saúde, a qualificação da gestão, dentre outros ${ }^{19}$.

A partir destas iniciativas do Ministério da Saúde e da Educação, o enfermeiro tem voltado, crescentemente, o seu foco de atenção para a educação, promoção e proteção da saúde. Nessa direção, os gestores, os usuários e os demais profissionais da saúde visualizam a atuação da enfermagem como algo considerado imprescindível, e que nos serviços há melhor desempenho quando estes são coordenados por enfermeiros. Para alguns autores, no entanto, o potencial do enfermeiro precisa ainda ser evidenciado, para um melhor aproveitamento da sua força de trabalho, podendo assim refletir na melhoria da qualidade da assistência prestada por esses profissionais junto aos serviços de saúde $e^{6}$.

No campo assistencial comunitário ou na ESF, o enfermeiro lança mão tanto de uma série de tecnologias que incluem os equipamentos e o instrumental necessários ao desenvolvimento do trabalho - como, por exemplo, os aparelhos, a estrutura física, os procedimentos técnicos, os folhetos educativos, os conhecimentos estruturados acerca da epidemiologia, o planejamento em saúde e outros -, quanto de tecnologias que envolvem as relações, as interações e as associações entre os usuários e as famílias, que dizem respeito ao vínculo, ao acolhimento, às relações humanizadas, dentre outros ${ }^{20}$. Nesse sentido, a atuação do enfermeiro vai além da dimensão técnico-assistencialista ou da aplicação imediata e direta dos conhecimentos técnico-científicos e se concentra em saberes que levam em consideração as inter-relações e a dinâmica coletivo-social de todos os envolvidos no processo.

No campo gerencial, o qual envolve o processo de trabalho da enfermagem na saúde coletiva, destacam-se as possibilidades de inserção na agenda municipal por meio das políticas públicas, as quais visam crescentemente atender tanto às necessidades de saúde da população, quanto considerar os níveis de satisfação profissional e pessoal dos trabalhadores. Deve-se considerar, no entanto, que o processo de trabalho ainda é marcado por diversos interesses, conflitos e necessidades, ou seja, por tensões e confrontos, nos quais, de um lado, se encontram os interesses políticos e, de outro lado, os interesses dos trabalhadores em geral, que nem sempre são equacionados de forma a atender aos anseios de ambas as partes envolvidas.

A partir do exposto, o enfermeiro não se apresenta neutro, como também não atua somente com saberes e práticas técnicas, ainda que necessite destas para constituir-se como prática social nos diferentes cenários da saúde. O espaço do enfermeiro, mais especificamente a partir do SUS e da ESF, se apresenta como um campo aberto e sensível às necessidades sociais emergentes. É evidente, porém, que esses esforços ainda são coroados por um êxito apenas modesto, considerando a necessidade de ampliar as discussões em torno do modelo de saúde, ainda hegemônico nos seus serviços e práticas, da formação de profissionais qualificados e comprometidos para o SUS e a ESF e, principalmente, da ampliação do debate acerca da enfermagem como prática social empreendedora.

\section{Considerações finais}

Um olhar retrospectivo sobre o papel do profissional enfermeiro no Sistema Único de Saúde brasileiro - 20 anos de SUS, permite argumentar que este e, de modo especial, a ESF, podem ser considerados estratégias facilitadoras e estimuladoras do processo de mobilização social, da ampliação da intervenção comunitário-coletiva, de um novo modo de pensar e agir, bem como pelas novas possibilidades interativas e associativas, à medida que sinalizam para uma nova abordagem de intervenção social, não mais focada nos reducionismos do saber médico-curativista, mas centrada na educação, promoção e proteção da saúde. Em outras palavras, à medida que, para superar o enfoque reducionista, os enfermeiros buscam adotar perspectivas integradoras de variáveis múltiplas, para captar amplamente a complexidade do processo saúde-doença.

Sendo uma profissão fundamental no sistema de saúde, a enfermagem se destaca e diferencia pelo desenvolvimento de práticas interativas e integradoras de cuidado, às quais vêm adquirindo uma repercussão cada vez maior, tanto na educação e promoção da saúde, quanto no fomento de políticas voltadas para o bem-estar social das famílias e comunidades. Nessa dire- 
ção, a enfermagem se configura, crescentemente, como a profissão do futuro, pela possibilidade de compreender o indivíduo não como um ser doente, mas como um ser singular e complexo, capaz de continuamente auto-organizar-se e projetar-se como autor do processo saúde-doença. Basta, no entanto, que a enfermagem invista em atitudes pró-ativas, capazes de promover e emancipar o indivíduo e a família como protagonistas da sua própria história.

Tendo o cuidado ao ser humano, em todas as suas dimensões, como essência e especificidade da profissão, a enfermagem tem a possibilidade de transitar pelos diferentes campos de conhecimento, bem como pelas diferentes realidades sociais. Tendo como foco a pessoa humana, a família e a comunidade, a enfermagem apresenta grande possibilidade de contribuir para a construção de um saber interdisciplinar, além de estabelecer canais efetivos de comunicação com os diversos setores sociais e, dessa forma, possibilitar estratégias mais eficazes e resolutivas de cuidado em saúde.

O papel do enfermeiro é reconhecido, em suma, pela capacidade e habilidade de compreender o ser humano como um todo, pela integralidade da assistência à saúde, pela capacidade de acolher e identificar-se com as necessidades e expectativas dos indivíduos e famílias, pela capacidade de acolher e compreender as diferenças sociais, bem como, pela capacidade de promover a interação e a associação entre os usuários, a equipe de saúde da família e a comunidade. A enfermagem se aproxima, se identifica e procura criar uma relação efetiva com o usuário, independentemente das suas condições econômicas, culturais ou sociais, ou seja, busca otimizar as intervenções de cuidado em saúde de modo que integre e contemple tanto os saberes profissionais quanto os saberes dos usuários e da comunidade.

\section{Colaboradores}

DS Backes trabalhou na concepção, delineamento e interpretação dos dados, MS Backes trabalhou na redação do artigo e interpretação dos dados, AL Erdmann trabalhou na pesquisa e provação da versão a ser publicada e A Buscher trabalhou na concepção, análise e revisão crítica do artigo. 


\section{Referências}

1. Backes DS. Viewing nursing care as a social enterprising practice [tese]. Florianópolis: Universidade Federal de Santa Catarina (UFSC); 2008.

2. Maurer FA, Smith CM. Community/Public Health Nursing Practice: Health for Families and Populations. $3^{a}$ ed. St. Louis: Elsevier Saunders; 2005.

3. Nies MA, Even M. Community Health Nursing. Promoting the Health of Population. St. Louis: Saunders Elsevier; 2007.

4. Schaeffer D, Buscher A, Ulrich R, Flöthmann J. Community Health Assessment fur Bielefeld-Schildesche. Veröffentlichungsreihe des Instituts fur Pflegewissenschaft. Bielefeld: Universität Bielefeld (IPW); 2008.

5. World Health Organization (WHO), Regional Office for Europe. Health 21: The health for all policy framework for the WHO European Region. Copenhagen: World Health Organization (WHO), Regional Office for Europe; 1999.

6. Barbosa MA, Medeiros M, Prado MA, Bachion MM, Brasil VV. Reflexões sobre o trabalho do enfermeiro em saúde coletiva. Rev Eletr Enfer 2004; 6(1):9-15.

7. Matumoto S, Fortuna CM, Mishima SM, Pereira MJB, Domingos NAM. Supervisão de equipes no Programa de Saúde da Família: reflexões acerca do desafio da produção de cuidados. Rev Inter-Comun Saúde Educ 2005; 8(16):9-24.

8. Alves VS. Um modelo de educação em saúde para o Programa Saúde da Família: pela integralidade da atenção e reorientação do modelo assistencial. Rev Inter-Comun Saúde Educ 2005; 9(16):39-52.

9. Costa GD, Cotta RMM, Ferreira MLM, Reis JR, Franceschini SCC. Family health: challenges in the reorientation process of the assistance model. Rev Bras Enfer 2009; 62(1):113-118.

10. Erdmann AL, Fernandes JV, Melo C, Carvalho BR, Menezes Q, Backes MTS. A visibilidade da profissão de enfermeiro: reconhecendo conquistas e lacunas. Rev Bras Enfer 2009; 62(4):637-643.

11. Matumoto S, Mishima SM, Pinto IC. Collective health: a challenge for nursing. Cad Saude Publica 2001; 17(1):233-241.
12. Brasil. Ministério da Saúde (MS). Constituição. República Federativa do Brasil. Brasília: Ministério da Saúde (MS); 1988.

13. Fleury S. Brazilian sanitary reform: dilemmas between the instituing and the institutionalized. Cien Saude Colet 2009; 14(3):743-752.

14. Brasil. Ministério da Saúde (MS). O desenvolvimento do Sistema Único de Saúde: avanços, desafios e rearfirmação dos seus princípios e diretrizes. Brasília: Ministério da Saúde (MS); 2002.

15. Strauss A, Corbin J. Basics of qualitative research: grounded theory - procedures and techniques. California: Sage Publication; 1990.

16. Strauss A, Corbin J. Pesquisa qualitativa: técnicas procedimentos para o desenvolvimento de teoria fundamentada. 2a ed. Porto Alegre: Artmed; 2008.

17. Carvalho YM, Ceccin RB. Formação e educação em saúde: aprendizados com a saúde coletiva. In: Campos GW, organizador. Tratado de Saúde Coletiva. Rio de Janeiro: Fundação Oswaldo Cruz (Fiocruz); 2007. p.232-241.

18. Silva FV. Autonomia profissional na enfermagem é construída por conquistas técnico-científicas, legais e pelo desenvolvimento de uma prática cidadã. Rev Bras Enfer 2007; 49(2):1-20.

19. Brasil. Ministério da Saúde (MS). Secretaria de Gestão do Trabalho e da Educação na Saúde-SG TES. Departamento de Gestão da Educação na Saúde-DEGES. Educação na saúde. Brasília: Ministério da Saúde (MS); 2006.

20. Falcón GCS, Erdmann AL, Backes DS. Meanings of care in health promotion. Rev Latino-Am. Enfer 2008; 16(3):419-424.

Artigo apresentado em 28/11/2009

Aprovado em 27/01/2010

Versão final apresentada em 15/02/2010 